\title{
EFFECT OF LOADING TIME ON THE STATIC FRICTION POLYMER-METAL SLIDING PAIRS AT LOW TEMPERATURE
}

\begin{abstract}
A. Ptak ${ }^{*}$
Abstract: Polymer materials working at low temperature significantly change their properties. This has a significant impact on their behavior during working with other elements. The goal of the present study was to analyze the influence of temperature on the static friction coefficient, which is important during the start-up of machine and equipment components. Thermoplastic materials: PA6, PEEK and PTFE were tested. These materials cooperated with typical C-45 structural steel using a pin-on-disc association. The sliding pairs were placed in a climatic chamber in which tests were carried out in the temperature range from $+20^{\circ} \mathrm{C}$ to $-50^{\circ} \mathrm{C}$. The obtained results allowed to determine the static friction coefficient for each sliding pair. The tests were supplemented with microscopic images of the surface and microhardness measurements.
\end{abstract}

Keywords: polymers, low temperature, static friction, tribology

\section{Introduction}

The friction process, although indispensable in nature as a scientific discipline, was not recognized until the second half of the 20th century. The study of friction, wear and lubrication, together with the accompanying phenomena in areas of solids or fluids pressed against with normal force, are referred to as tribology. The discovery of a way to completely overcome the effects of friction between two cooperating surfaces would mean a big breakthrough in science. It is estimated that about one-third of the world's energy resources are used to overcome the frictional resistance (Hebda, 2007). In addition, the natural consequence of friction is wear, it should be prevented to avoid frequent replacement of components, assemblies or entire machines (e.g. by oxidation of the surface layer (Aniolek et al., 2016)). If wear occurs and it is necessary to replace parts, it have to be identify the problem that caused wear to prevent it in the future using appropriate techniques (Sokolski, 2017, Feldshtein et al., 2014).

However, it should be remembered that there would be no technical progress without friction. There would be no movement or even life. All organisms, as well as land, water and air vehicles move as a result of friction. Tribology does not aim to avoid friction but prediction its value and quality.

The tribological processes at low temperature are an issue that is particularly rarely discusses in the literature. Low, i.e. from $-50{ }^{\circ} \mathrm{C}$ to $0{ }^{\circ} \mathrm{C}$, because research within the absolute zero temperature is a very common topic of the dissertations concerning machines and vehicles operating in a vacuum (Gradt et al., 1998, Ostrovskaya et al., 2001, Thelier et al., 2010). One of these issues is static friction in polymer-metal nodes. Due to the significant changes in polymer properties depending on the temperature, these nodes require special treatment. In addition, the topic is difficult to generalize, because even slightly different structure polymers may significantly differ in mechanical, chemical or thermal properties (Landel et al., 1993, Hartwig, 1994). For this reason, most of them require additional research and an individual approach to the problem.

Anita Ptak, PhD.: Department of Fundamentals of Machine Design and Tribology, Faculty of Mechanical Engineering, Wroclaw University of Science and Technology, Wybrzeze Wyspianskiego 27; 50-370, Wroclaw; PL, anita.ptak@pwr.edu.pl 


\section{Methodology}

In order to measure the resistance to motion caused by frictional force, a "pin-on-disc" test stand was used. The stand was placed in a climatic chamber, which allowed testing in the temperature range from $\mathrm{T}=-70{ }^{\circ} \mathrm{C}$ to $\mathrm{T}=+150{ }^{\circ} \mathrm{C}$ (Fig. 1). The change in temperature helps in making measurements in various environmental conditions.

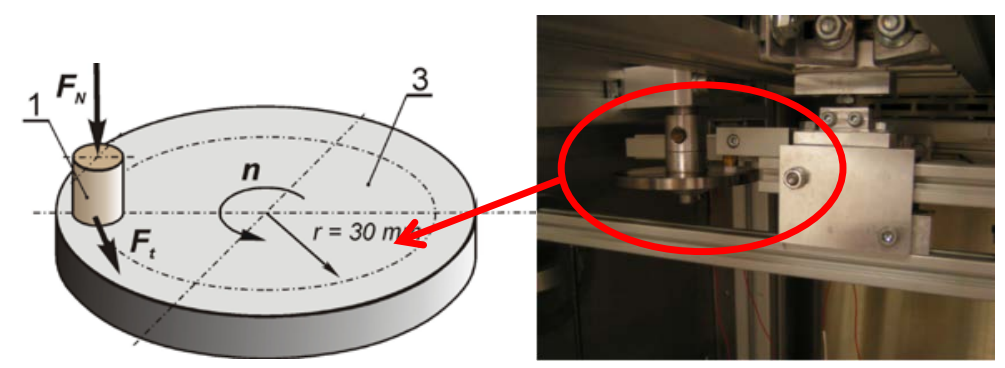

Fig. 1: Pin-on-disc test stand: diagram and picture in the climatic chamber.

Three types of thermoplastic polymers were selected for tribological tests, which cooperated with one steel type. The selection criterion was the frequency of using unmodified polymers in the slip pairs of machines and devices: PA6 (polyamide), PEEK (polyetheretherketone) and PTFE (polytetrafluoroethylene). The metal counterspecimen was construction steel of higher quality C45 with roughness $R_{a}=0.3 \mu \mathrm{m}$ and hardness 45 HRC.

The experiment was made in the same conditions for all sliding pairs. Five temperature values were assumed below $0{ }^{\circ} \mathrm{C}$ and for comparison purposes also at $0{ }^{\circ} \mathrm{C}$ and $20^{\circ} \mathrm{C}$. Fixed values of loading time were also determined, the following five values were adopted: $30 \mathrm{~s}, 60$ s, 120 s, $180 \mathrm{~s}$ and $300 \mathrm{~s}$. During the cooling of the inside of the climatic chamber, the polymer sample was constantly loaded with a force $\mathrm{N}=9.81 \mathrm{~N}$ and the steel disc was moving at the lowest possible speed. That prevented freezing the sample into the disc. The registration of the friction force was made during the slow acceleration of the disc to the rotational speed $n=200 \mathrm{rpm}$ with a step $t=0.02 \mathrm{~s}$. Each of the measurements repeated three times. All obtained results were subjected to statistical calculations. The exact test program is shown in the Figure 2. The tribological tests were supplemented by a complex instrumental study. Both before the tribological tests and after them, the top layer condition was assessed by means the microhardness parameters and microscopic observations (using SEM - Scanning Electron Microscope).

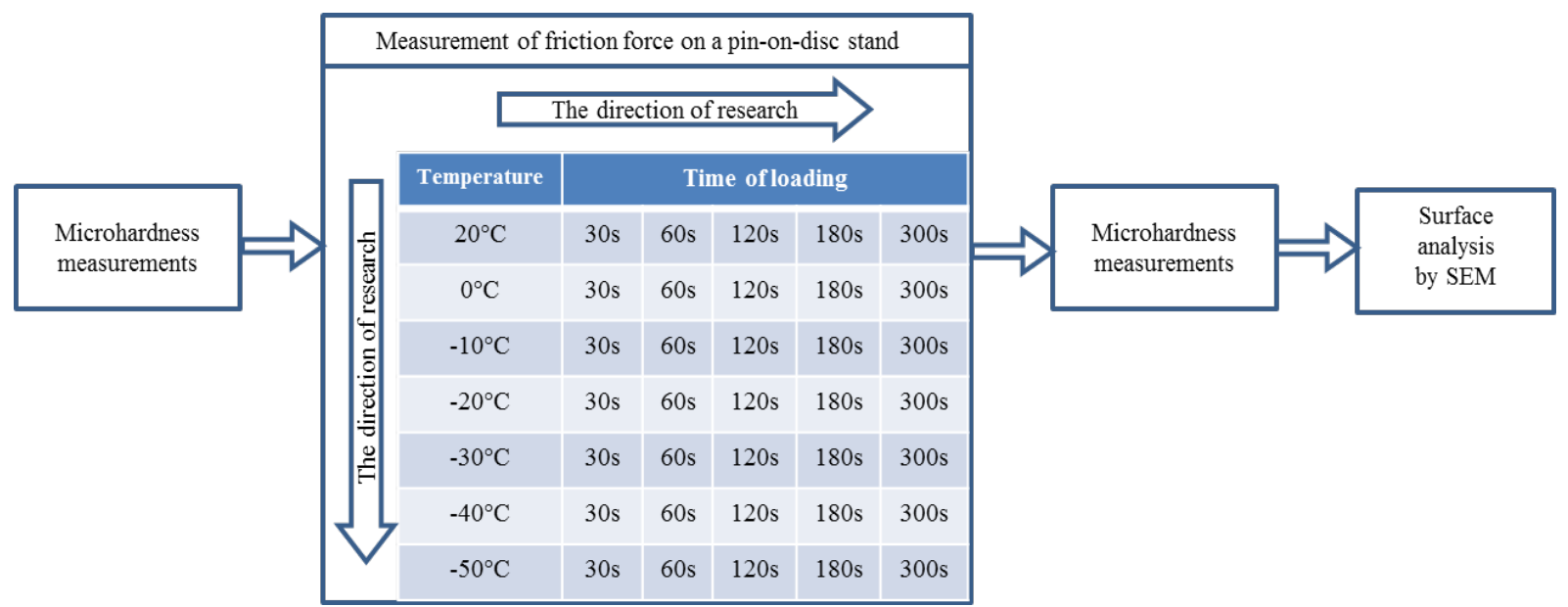

Fig. 2: Diagram of the tribological research at low temperature.

\section{Results and discussion}

During tribological experiments a maximum friction force at which the contact of the tested materials was breaking was observed. The static friction coefficient was calculated using the appropriate formulas. All results and static calculations were present in the diagrams and tables included in this paper. The research results are shown in Figure 3, whereas supplementary research results are presented in Table 1 and selected diagrams illustrating the surface layer parameters change are presented in Figure 4. 
Table 1 presents the results of microhardness measurements of the sample at room temperature (before tribological measurements) and directly removed from the chamber at $-50{ }^{\circ} \mathrm{C}$ (after tribological measurements).
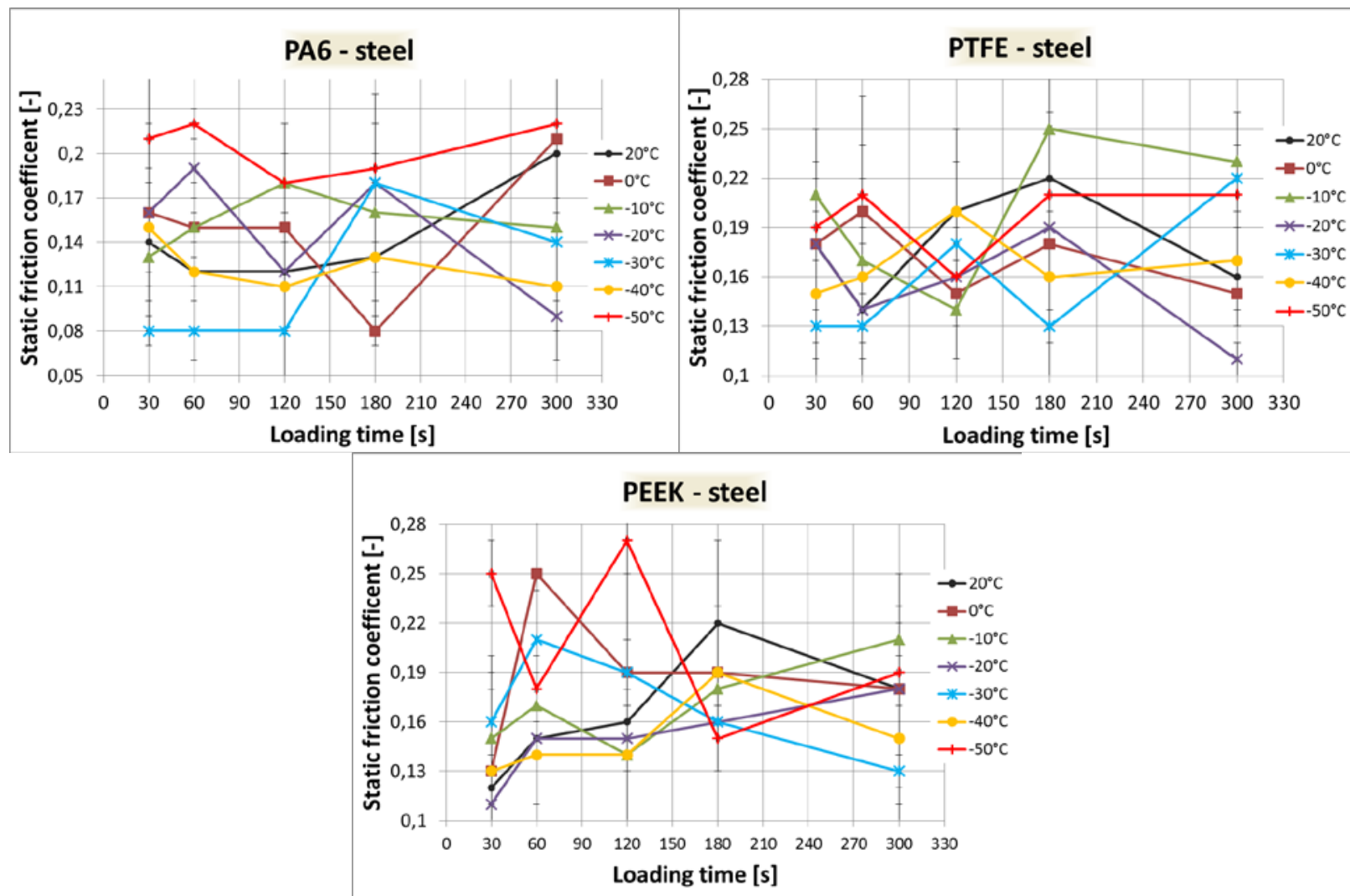

Fig. 3: The influence of temperature on the static friction coefficient depending on the loading time for all sliding pairs:metal with PA6, PTFE and PEEK.

Tab. 1: Results of microhardness measurements of polymer samples for different temperatures.

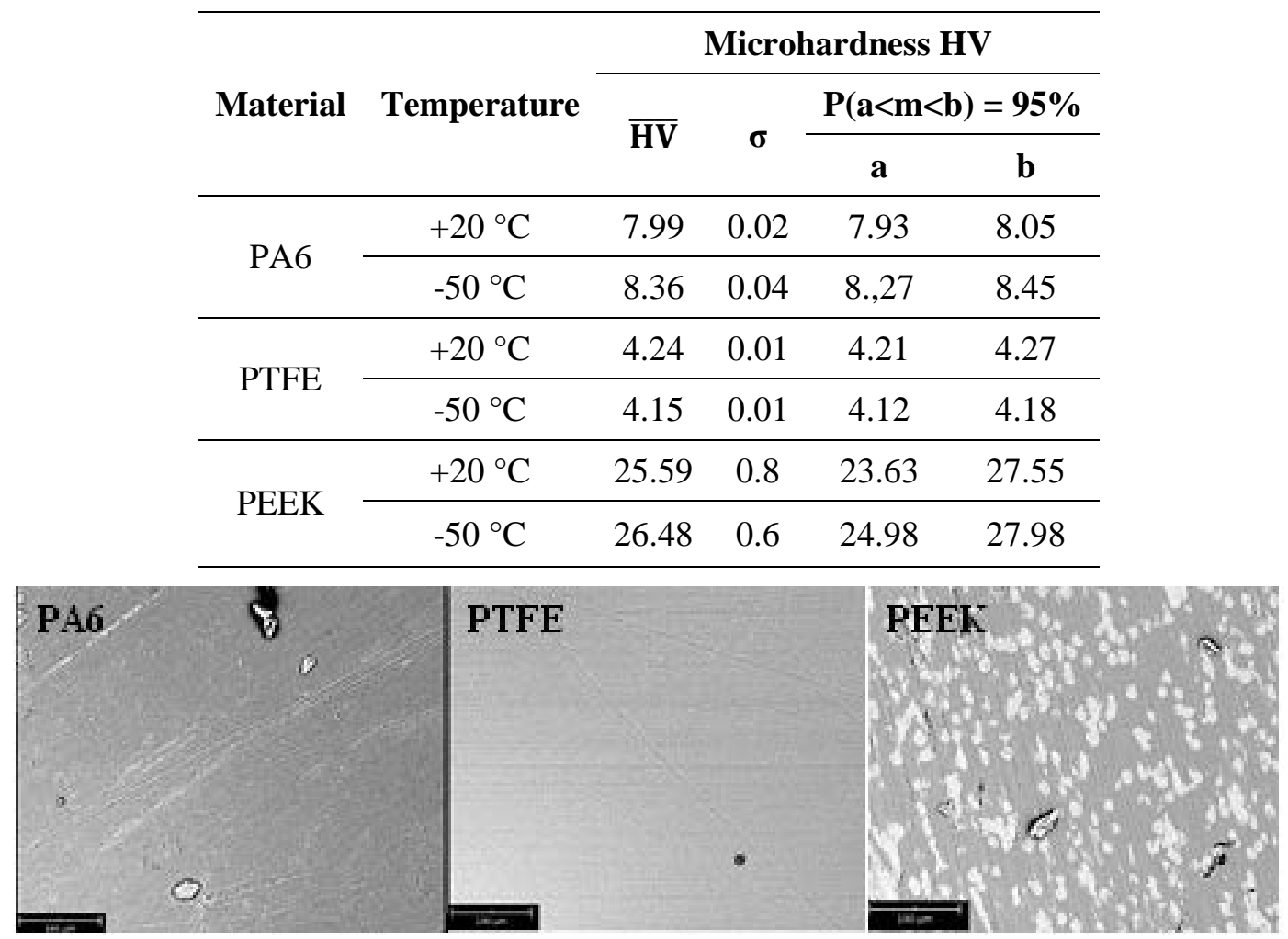

Fig. 4: Selected SEM microscopic images after tribological tests. 
The tribological research has shown that it is difficult to determine the close relationship between the static friction coefficient and the temperature and time of loading. The obtained characteristics allow to conclude that for PA6 and PEEK as the temperature was reduced, more unstable characteristics were obtained. For PTFE, however, it is clearly visible that as the temperature decreases, the time of loading has less and less influence on the measurement result. Approximately the characteristics are increasing nature, i.e. the static friction coefficient increases with the increase of the loading time. It may be related to the phenomenon of adhesion, which also takes place at a temperature below $0{ }^{\circ} \mathrm{C}$ (according to the conducted tests). For all materials, the reduction in temperature was the reason for the curing of the surface layer manifested by an increase in the microhardness of the samples. Microscopic images showed the presence of harder particles being the wear products created during the cooperation of elements. These particles may have formed during the stabilization of temperature, because the movement was then long-lasting. Products of wear could have affected the unstable results of static friction tests.

\section{Summary}

The tribology of thermoplastic polymers is a highly complex issue. The steel - polymer sliding node is very susceptible to external factors, especially at low temperature. In addition, each of the plastics is characterized by different mechanical properties (e.g. microhardness) and thermal properties (e.g. softening and crushing temperature), which have a decisive influence on friction parameters. The result is that the preparation of universal characteristics with special points becomes an extremely difficult and time-consuming task.

While at room temperature it has been repeatedly confirmed that the static loading time of the sample affects its static coefficient of friction, the topic of low temperature has not been sufficiently investigated. It is supposed that this is due to the weakening phenomenon of creep, what is not excluded by the research carried out in this work. Despite local increases in the static coefficient of friction as a function of loading time for low temperature, the characteristics drawn up do not show clearly increasing or decreasing tendencies.

PEEK is characterized by characteristics with the most stable results, which is why further research would be good to start with this material. It would also be advisable to study the short loading times, because the properties of the logarithmic function indicate that changes in this range may be the most intense. The parameter having a significant impact on the measurement results can be the value of the loading force, which (like the loading time) determines the actual contact surface in the friction nodes.

\section{References}

Aniolek K., Kupka M. and Barylski A. (2016) Sliding wear resistance of oxide layers formed on a titanium surface during thermal oxidation, Wear, Volumes 356-357, 15 June 2016, Pages 23-29.

Feldshtein E., Kowalewski P. and Dyachkova L. (2014) Studying the surface layers of products using a low-vacuum scanning electron microscope. Journal of Surface Investigation : X-ray, Synchrotron and Neutron Techniques. 2014, vol. 8, nr 6, s. 1258-1264.

Gradt T., Schneider T., Hübner W. and Börner H. (1998) Friction and wear at low temperatures. International Journal of Hydrogen Energy 23, 5, 397-403.

Hartwig G. (1994) Polymer properties at room and cryogenic temperatures. Springer.

Hebda M. (2007) Processes of friction, lubrication and wear of machine, Publisher of the Institute for Sustainable Technologies, Warsaw.

Landel R. F. and Nielsen L. E. (1993) Mechanical properties of polymers and composites. CRC Press.

Ostrovskaya Y. L., Yukhno T. P., Gamulya G. D., Vvedenskij Y. V. and Kuleba V. I. (2001) Low temperature tribology at the B. Verkin Institute for Low Temperature Physics \& Engineering (historical review). Tribology International 34, 4, 265-276.

Sokolski P. (2017) On wear processes in pin joints in caterpillars of large-size working machines, Engineering Mechanics 2017, Brno University of Technology, Brno, pp. 910-913.

Theiler G. and Gradt T. (2010) Friction and wear of PEEK composites in vacuum environment. Wear 269, 3-4, 278-284. 